\title{
Predicting Hyperkalemia by a Two-Staged Artificial Neural Network
}

\author{
MF Wu, JJ Chiang ${ }^{1,2}$, YC Yang, IH Chao, SM Shieh ${ }^{2}$, WC Tzeng, JC Hsieh \\ ${ }^{1}$ Department of Bioinformatics, Chung Hua University, Hsin-Chu, Taiwan \\ ${ }^{2}$ Wei Gong Memorial Hospital, Miao-Li, Taiwan
}

\begin{abstract}
Hyperkalemia-induced arrhythmias are commonly observed in emergency medicine. Clinically, hyperkalemia is verified through laboratory tests, which takes 20 minutes or more to generate results. To increase the efficiency of treatment, the identification and classification of hyperkalemia directly from electrocardiogram are crucial. In this study, we developed a two-stage artificial neural network (ANN) to classify the disorder of electrolyte from 20 normal and 20 moderate hyperkalemic (5.4 -7.4 mmole/L) individuals. The first stage of ANN, consisting of two multi-layer back-propagation ANNs, was fed with selected features extracted directly from 12-lead electrocardiograms. The second stage was composed of a two-layer backpropagation ANN receiving input from the outcomes of previous stage. A total of 17 features were selected, among which 12 features including $T$ wave amplitude and duration from V1 to V6 leads served as the inputs of the first ANN in the first stage and the other 5 features including $P$ wave amplitude and duration, QRS duration, $P R$ interval, averaged RR interval from lead II served as the inputs of the second $A N N$ in the first stage. By training the two-stage ANN with 30 normal and 30 hyperkalemic cases, the results indicated that the accuracy is $62.5 \%$ with sensitivity 12/20. In conclusion, the algorithm developed in this study can predict hyperkalemia more efficiently compared to the prediction by experienced clinicians whose accuracy is $50 \%$ with sensitivity $7 / 20$. It can be expected that the sensitivity and the accuracy will be promoted once the training data increase.
\end{abstract}

\section{Introduction}

Hyperkalemia was seen commonly in the department of emergency medicine in clinics. Hyperkalemia can be degenerated into cardiac fibrillation soon and leads to death if it is not diagnosed in time. Traditionally, the procedure for diagnosis fea hyperkalemia is through the laboratory blood test which will take 20 minutes or more. In order to increasing the efficiency of diagnosis and therapeutic timing, a non-invasive method to diagnose hyperkalemia is crucial. In clinics, 12-lead electrocardiogram (ECG) was usually used to predict the possibility of hyperkalemia. However, the accuracy is very low (about $50 \%$ ) even by experienced cardiologist [1, 2]. Because the ECG features in response to hyperkalemia are not obvious. Based on clinical reports [1,2], hyperkalemia was associated with the change of ECG by some characteristics. For examples, peaked $\mathrm{T}$ waves and widen $T$ waves could be observed in some chest leads during mild hyperkalemia, and flatten $\mathrm{P}$ waves in some limb and chest leads. Other vulnerable variable such as increased PR interval, QRS interval, and RR interval could be seen during mild to severe hyperkalemia $[2,3]$. However, the choice of exact features in response to hyperkalemia is difficult because of the variability of features. Recent development of artificial neural networks had been implemented on the applications of medicine and had successful evidences showing the ability on pattern recognitions [4]. The applications of neural networks on the detection of cardiovascular diseases had big progress such as myocardial infarctions and atrial fibrillation [5]. Recent Poter etc [1] used successfully traditional back-propagation neural networks to detect severe hyperkalemia with a sensitivity $89 \%$ by single lead II ECG signals. However, the hyperkalemic data were collected by controlled conditions. In Poter's experimental dog model, qualified data were limited by some conditions such as absent $\mathrm{P}$ wave, decreased ventricular rate $>20 \%$ in 5 minutes under $2 \mathrm{mEq} / \mathrm{kg} / \mathrm{hr}$ of potassium chloride was given and potassium concentration must be great than $7.7 \mathrm{mmole} / \mathrm{L}$. In this study, we try to extract possible features from 12-lead ECG signals, and construct a neural network to predict hyperkalemia from light to severe conditions.

\section{Methods}

\subsection{Paper ECG processing}

The 50 cases of 12-lead paper ECG patient records whose diagnosis were hyperkalemia ranged from $5.4 \mathrm{mmole} / \mathrm{L}$ to $9.6 \mathrm{mmole} / \mathrm{L}$ were collected by the department of emergency medicine at Wei Gong memorial hospital 
from October, 2002 to March, 2003. The paper ECG records were scanned by 600 dpi resolution and saved as image files. The pixels of ECG trace on the image files were collected and re-sampled using a fixed $200 \mathrm{~Hz}$ rate by linear interpolation. After digitalization of paper ECG, the baseline of ECG was correction and unwanted artifacts were removed. Digitalized ECG data were ready for extraction of possible physiological parameters.

\subsection{Features selection}

Based on the communication with clinical physicians, some possible features showing on ECG during hyperkalemia were summarized in table-1.

Table -1 Features of Hyperkalemia

\begin{tabular}{|l|l|}
\hline \multicolumn{1}{|c|}{ Features } & $\begin{array}{l}\text { Potassium } \\
\text { concentration }\end{array}$ \\
\hline Flatten P waves on limb leads & $5.5-6.5 \mathrm{mmole} / \mathrm{L}$ \\
\hline $\begin{array}{l}\text { Peaked T wave on chest leads } \\
\text { Increased PR interval, } \\
\text { Increased QTc }\end{array}$ & $6.6-8 \mathrm{mmole} / \mathrm{L}$ \\
\hline Widen QRS complex & Above $8 \mathrm{mmole} / \mathrm{L}$ \\
\hline
\end{tabular}

In light hyperkalemia, ECG on limb leads could have showed decreased amplitude and increased duration in $\mathrm{P}$ waves. We selected the amplitude and duration of $P$ wave in lead II as the input parameters. Because of increased potassium concentration, the decreased conduction can lead to increased PR interval and QTc. In severe hyperkalemia, a sine wave is usually observed because widen QRS complex could merge with $\mathrm{P}$ wave. Totally, 5 features including the amplitude and duration of $\mathrm{P}$ wave in lead II , PR interval, QTc, and QRS complex width are selected as the input parameters of the first stage of neural network. In clinics, physicians usually predict the hyperkalemia by the occur of peaked $\mathrm{T}$ waves in chest leads. It is the most important index to predict hyperkalemia for clinical physicians. Obviously, peaked $\mathrm{T}$ waves were observed easily in all hyperkalemic cases. We select 12 features composed of the amplitude and duration of $\mathrm{T}$ wave in v1 to $\mathrm{v} 6$ six chest leads to be the input parameters of the second stage of neural networks.

\subsection{A two-stage neural network}

A two staged back propagation neural network was developed to predict hyperkalemia. Each stage is serial together and had the same structure. The neural network is composed of a 100-neuron hidden layer and a oneneuron output layer. The activation functions in hidden layer and output layer were expressed as shown in equation (1) and equation (2):

$$
\begin{aligned}
& f(n 1)=\frac{\exp (n 1)-\exp (-n 1)}{\exp (n 1)+\exp (-n 1)} \\
& f(n 2)=\frac{1}{1+\exp (-n 2)}
\end{aligned}
$$

Where $\mathrm{n} 1$ represented computed values by weighting input parameters, and $\mathrm{n} 2$ represented the values after. the weighting output of hidden neurons.

The structure of the two staged neural network was shown in figure 1. 12 selected parameters from chest leads were fed into the first staged neural network. The output of the first stage network was as one input of the second staged neural network.

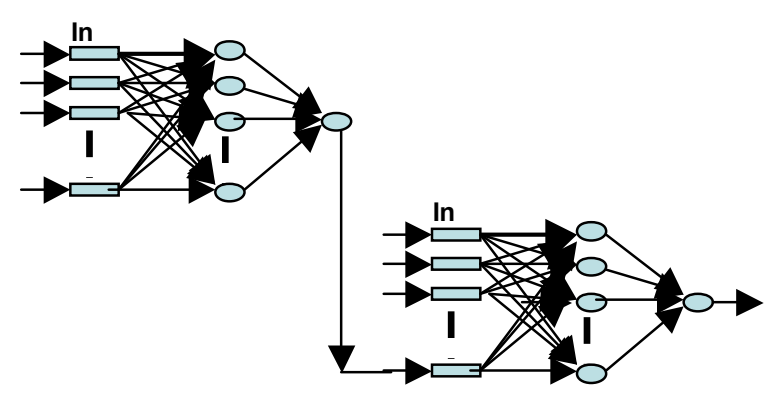

Figure 1: The structure of a two-staged neural network.

\section{Results}

Table -2 The Results of Predictive Hyperkalemia

\begin{tabular}{|c|c|}
\hline Sensitivity & Specifity \\
\hline $60 \%$ & $65 \%$ \\
\hline
\end{tabular}

By training the two-stage ANN with 30 normal and 30 hyperkalemic cases, the results indicated that the total accuracy is $62.5 \%$ with sensitivity $60 \%$ and specifity $65 \%$.

\section{Conclusions}

The ECG features which were responsible for hyperkalemia were uncertain and were not elucidated. In this study, we selected 17 possible features which were observed often in clinical hyperkalemic cases. For reducing the complexity in pattern recognition, some other features such as ST elevation/depression, S-T interval, and P-R segment were ignored. In order to compare the efficiency of the two-staged network, a experienced physicians at department of emergency 
medicine joined the test of hyperkalemia by ECG directly. In conclusion, the algorithm developed in this study can predict hyperkalemia more efficiently compared to the prediction by experienced clinicians whose accuracy is $50 \%$ with sensitivity $7 / 20$. It can be expected that the sensitivity and the accuracy will be promoted once the training data increase.

\section{References}

[1] Poters RS etc. Prediction of hyperkalemia in dogs from electrocardiographic parameters using an artifificial neural network. Academic Emergency Medicine. 2001; 8(6):599603.

[2] Wrenn KD etc. The ability of physicians to predict hyperkalemicnfrom the ECG. Ann Emerg Med. 1991; 20:1229-1232.

[3] Dreifus LS etc. A clinical correlative study of the electrocardiogram in electrolyte imbalance. Circulation. 1956; 14: 815-825.

[4] Penny W etc. Neural networks in clinical medicine. Med Decis Making. 1996; 16:386-398.

[5] Yang TF etc. Use of artificial neural networks within deterministic logic for the computer Eferior myocardial infarction. Journal of Electrocardiology 1994; 27:188-193.

\section{Acknowledgements}

This study was sponsored by NSC 92-2213-E-216-012.

Address for correspondence.

Jui-chien Hsieh, Ph.D.

Department of Bioinformatics

Chung Hua University

30 Tung Shiang, Hsin-chu 30067

Taiwan

E-mail address : jchsieh@chu.edu.tw 